\title{
Prognostic implications of intratumoral CD103+ tumor-infiltrating Iymphocytes in pulmonary squamous cell carcinoma
}

\author{
Jaemoon Koh ${ }^{1,2}$, Sehui Kim ${ }^{1}$, Moon-Young $\mathrm{Kim}^{3}$, Heounjeong $\mathbf{G o}^{4}$, Yoon Kyung \\ Jeon ${ }^{1,5, *}$, Doo Hyun Chung ${ }^{1,2,6, *}$ \\ ${ }^{1}$ Department of Pathology, Seoul National University Hospital, Seoul National University College of Medicine, Seoul, Republic \\ of Korea \\ ${ }^{2}$ Department of Biomedical Sciences, Seoul National University College of Medicine, Seoul, Republic of Korea \\ ${ }^{3}$ Department of Forensic Medicine, Seoul National University College of Medicine, Seoul, Republic of Korea \\ ${ }^{4}$ Department of Pathology, Asan Medical Center, University of Ulsan College of Medicine, Seoul, Republic of Korea \\ ${ }^{5}$ Tumor Immunity Medical Research Center, Tumor Microenvironment Global Core Research Center, Cancer Research Center, \\ Seoul National University College of Medicine, Seoul, Republic of Korea \\ ${ }^{6}$ Ischemic/Hypoxia Institute, Seoul National University College of Medicine, Seoul, Republic of Korea \\ *These authors contributed equally to this work
}

Correspondence to: Doo Hyun Chung, email: doohyun@snu.ac.kr Yoon Kyung Jeon, email: junarplus@chol.com

Keywords: CD103, tumor-infiltrating lymphocytes, pulmonary squamous cell carcinoma, prognosis, cancer immunotherapy Received: August 01, 2016

Accepted: January 06, 2017

Published: January 13, 2017

\section{ABSTRACT}

CD103 is the $a_{E}$ subunit of $a_{E} \beta_{7}$ integrin that is expressed in tissue-resident memory $T$ cells, where it promotes cytotoxic $T$ cell responses against tumors. However, little is known about its expression or clinicopathological implications in non-small cell lung cancer (NSCLC). This study investigated the prognostic implications of CD103+ tumor-infiltrating lymphocytes (TILs) in NSCLC. We established two cohorts: patients with resected NSCLC $(n=132)$ and patients with pulmonary squamous cell carcinoma (pSCC), a subset of NSCLC $(n=378)$, to estimate the prognostic significance of $\mathrm{CD}_{103}{ }^{+}$TILs. The numbers of $\mathrm{CD} 103^{+}$TILs in the intratumoral (i.e., intraepithelial) and stromal regions of NSCLC were estimated using immunohistochemistry and automated image analysis. In the NSCLC cohort, high numbers of intratumoral CD103 ${ }^{+}$TILs were significantly associated with prolonged disease-free survival (DFS) and overall survival (OS) in patients with pSCC but not in those with pulmonary adenocarcinoma. In the pSCC cohort, a positive correlation was observed between the numbers of intratumoral $\mathrm{CD}_{103^{+}}$and $\mathrm{CD8}{ }^{+}$TILs (correlation coefficient $=0.736, P<0.001$ ). The ratio of intratumoral/stromal $\mathrm{CD}_{103^{+}}$TILs was higher in pSCC with high compared to low E-cadherin expression $(P=0.021)$. According to Kaplan-Meier analysis, high intratumoral but not stromal $\mathrm{CD}_{103}{ }^{+}$TILs were associated with prolonged DFS and OS in patients with resected pSCC $(P=0.021$ and 0.002 , respectively $)$. Multivariate analysis revealed that a high number of intratumoral CD103 ${ }^{+}$TILs is an independent predictor of a more favorable DFS $(P=\mathbf{0 . 0 2 1})$. Thus, a high number of intratumoral $\mathrm{CD}_{103^{+}}$TILs is a favorable prognostic indicator in patients with pSCC.

\section{INTRODUCTION}

In recent decades, therapies targeting driver gene mutations in tumors have significantly improved the survival of patients with non-small cell lung cancer (NSCLC) [1]. Among the different types of NSCLC, druggable genetic changes have been variably observed in pulmonary adenocarcinoma (pADC), whereas these mutations are rarely found in pulmonary squamous cell carcinoma (pSCC). Moreover, acquired resistance to targeted therapy eventually develops via diverse mechanisms in almost all treated patients [2-4]. Thus, 
novel therapeutic strategies are needed to overcome these limitations in NSCLC.

Tumor surveillance involves dynamic interactions between tumor cells and variable cell components in the microenvironment, which are also targeted by cancer control strategies [5]. The tumor microenvironment is composed of heterogeneous cells including immune cells, endothelial cells, and fibroblasts. The immune system is critical for regulating tumor development and progression by balancing pro-inflammatory and anti-inflammatory responses [6]. Recently, several immune checkpoints such as the programmed cell death (PD)-1/PD-ligand 1 (PD-L1) and CTLA4 pathways have emerged as immunotherapy targets for solid tumors, particularly NSCLC, thereby improving patient survival [7]. Thus, a comprehensive understanding of immune cell network interactions in the tumor microenvironment is important in clinical oncology.

$\mathrm{CD}^{+}$cytotoxic $\mathrm{T}$ lymphocytes are critical effector cells in adaptive immunity involved in killing tumor cells [8]. Moreover, many studies have demonstrated that high numbers of $\mathrm{CD}^{+}$tumor-infiltrating lymphocytes (TILs) are significantly correlated with prolonged survival in patients with various cancers, including bladder, ovarian, and lung cancers [9-12]. Furthermore, interactions between tumor and immune cells via cell adhesion molecules are critical for maintaining immune responses against tumor cells [13]. Among the adhesion molecules, CD103 is the $\alpha_{E}$ subunit of the heterodimeric $\alpha_{E} \beta_{7}$ integrin that mediates cell adhesion, migration, and signaling via interaction with its ligand, E-cadherin, expressed in epithelial cells [14]. Under homeostasis, CD103 ${ }^{+}$ expression in memory $\mathrm{T}$ cells residing in peripheral tissues, including the skin and mucosa, increases the migration and retention of these cells $[15,16]$. Moreover, the interaction between CD103 and E-cadherin is responsible for the recruitment and retention of antigenspecific TILs in gliomas, and ovarian cancer, and lung cancer [17-19]. CD103 expression was observed more frequently in the TILs of intratumoral (i.e., intraepithelial) than stromal regions, and this has been implicated in patient survival [20-22].

Recently, high numbers of CD103+ TILs were correlated with improved survival in patients with early stage NSCLC [20]. However, their prognostic implications in patients with NSCLC according to histological subtype and localization remain unclear. Thus, we investigated the expression pattern of CD103 in TILs according to the clinicopathological characteristics of patients with NSCLC.

\section{RESULTS}

\section{Immunohistochemical analysis of intratumoral CD103 $^{+}$TILs in NSCLC}

To investigate the potential prognostic value of intratumoral $\mathrm{CD}_{103}{ }^{+}$TILs in NSCLC, we performed immunohistochemistry (IHC) analysis of CD103 in the NSCLC cohort $(n=132)$. The mean numbers of intratumoral and stromal $\mathrm{CD}_{103}{ }^{+} \mathrm{TILs} / \mathrm{mm}^{2}$ were $357.5 \pm 368.9$ and $225.0 \pm 205.5$ in patients with pSCC $(n=47)$ and $182.0 \pm 220.9$ and $233.0 \pm 269.2$ in patients with pADC $(n=85)$, respectively (Figure 1A and 1B). The correlation between the intratumoral CD103 ${ }^{+} \mathrm{TIL}$ number and the clinicopathologic features of patients with NSCLC are summarized according to histological subtype in Supplementary Table 1 . High numbers of CD103 ${ }^{+}$TILs were correlated with male sex $(P=0.051)$ and smoking status $(P=0.023)$ in patients with ADC. Smokers exhibited higher numbers of intratumoral CD103 ${ }^{+}$TILs compared to non-smokers among the patients with pSCC; however, this difference was statistically insignificant, potentially due to the skewed population of non-smokers $(n=2)$ with pSCC. Kaplan-Meier survival analysis revealed a longer disease-free survival (DFS) $(P=0.038)$ and overall survival (OS) $(P=0.038)$ among patients with high intratumoral

\section{A Low numbers of Intratumoral $\mathrm{CD} \mathrm{A3}^{+} \mathrm{TILS}^{-}$}

Squamous cell carcinoma

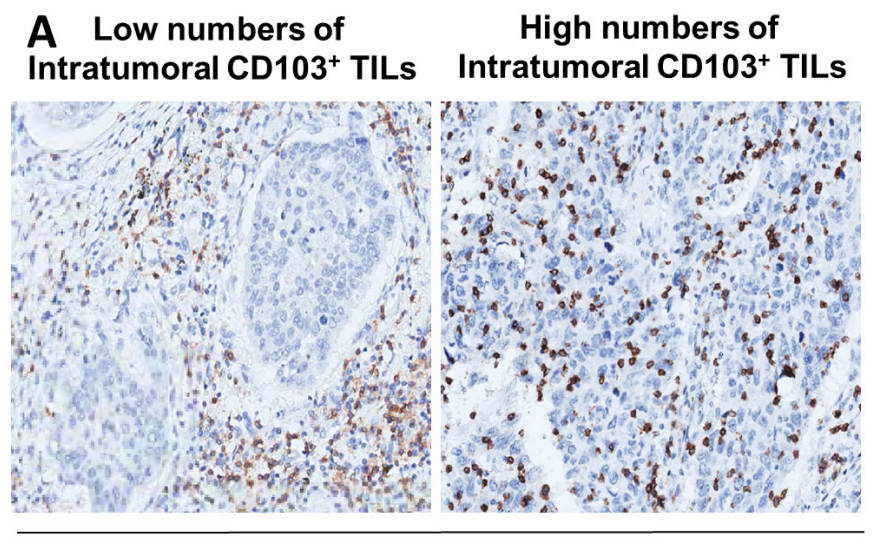

\section{B Low numbers of Intratumoral CD103+ ${ }^{+}$ILs Intratumoral CD103+ ${ }^{+}$ILs}

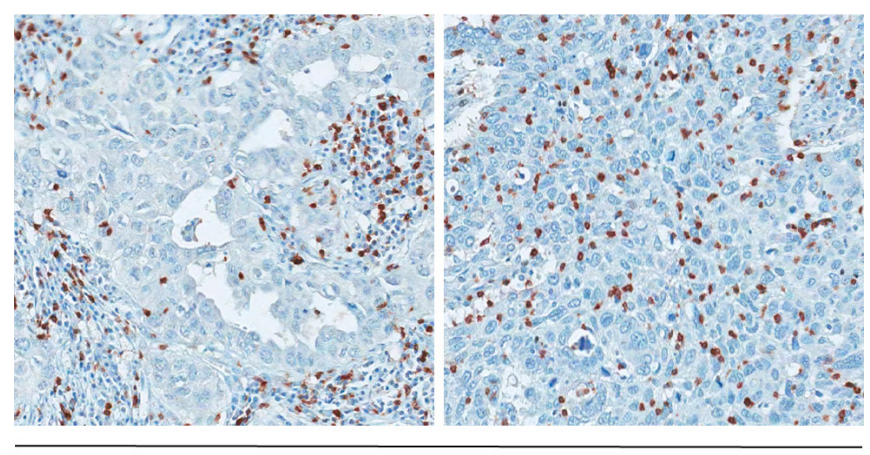

Adenocarcinoma

Figure 1: Representative immunohistochemical images of low versus high $\mathrm{CD} 103^{+}$cell numbers in intratumoral regions of pulmonary squamous cell carcinoma (A) and adenocarcinoma (B). 
$\mathrm{CD}_{103^{+}}$TIL numbers in pSCC (Supplementary Figure 1A). However, the number of intratumoral CD $103^{+}$TILs was not significantly related to DFS or OS in patients with pADC (Supplementary Figure 1B).

\section{Comparative analysis of $\mathrm{CD} 103^{+}$and $\mathrm{CD8}^{+}$TIL numbers in pSCCs according to clinicopathologic features}

Based on the CD103+ TIL results in the NSCLC cohort, we postulated that $\mathrm{CD} 103^{+}$TILs are significantly correlated with survival of patients with pSCC. To address this further, we established a cohort comprising a large number of patients with pSCC $(n=378)$. Several recent lines of evidence suggest that $\mathrm{CD} 8^{+} \mathrm{CD} 103^{+}$TILs account for the majority of CD103 ${ }^{+}$TILs $[18,20,23]$. Thus, we analyzed CD8 and CD103 expression in TILs of pSCC intratumoral and stromal regions. In the intratumoral regions, the number of $\mathrm{CD}_{103^{+}}$TILs was higher than that of $\mathrm{CD}^{+}$TILs $(222.2 \pm 299.0$ and $175.0 \pm 268.8$, respectively; $P<0.001$ ), whereas in the stromal regions, the number of $\mathrm{CD}^{+}$TILs was much higher than that of $\mathrm{CD} 103^{+}$TILs in stromal areas $(173.2 \pm 175.4$ and $108.7 \pm 185.0$, respectively; $P<0.001)$. However, the numbers of $\mathrm{CD} 103^{+}$and $\mathrm{CD}^{+}$TILs were positively correlated in intratumoral regions $(\mathrm{r}=0.736, P<0.001$, as shown in Figure 2). Moreover, the clinicopathological characteristics of patients with pSCC were evaluated with respect to the numbers of $\mathrm{CD} 103^{+}$and $\mathrm{CD} 8^{+}$TILs in intratumoral and stromal regions (Table 1). Tumor size was inversely correlated with the number of intratumoral $\mathrm{CD}_{103^{+}}$TILs $(P=0.037)$, whereas smokers exhibited significantly high numbers of stromal CD103 ${ }^{+}(P<0.001)$ and $\mathrm{CD}^{+}$TILs $(P=0.001)$ in the pSCC cohort.

\section{Relationship between intratumoral $\mathrm{CD} 103^{+} \mathrm{TIL}$ numbers and $\mathrm{E}$-cadherin expression in pSCC tumor cells}

Several studies have demonstrated that the interaction between E-cadherin expressed on epithelial cells and CD103 expressed on lymphocytes plays an important role in the retention of antigen-specific lymphocytes within epithelial tissue [17-19]. Thus, we analyzed and compared E-cadherin expression in tumor cells with respect to the number of intratumoral CD $103^{+}$TILs. The expression of E-cadherin was higher in patients with pSCC (58.3\%) compared to those with pADC (29.7\%) in NSCLC cohort (Supplementary Table 1) and the H-score of E-cadherin was higher in patients with pSCC (mean, 92.8) than in those with pADC (mean, 52.1) among NSCLC cohort. In pSCC cohort, the mean H-score of E-cadherin is 130.0.

The number of intratumoral CD $103^{+}$TILs appeared to be higher in pSCC positive for E-cadherin than $\mathrm{pSCC}$ negative for E-cadherin expression, but this result was statistically insignificant (Supplementary Table 1). The numbers of intratumoral CD $103^{+}$TILs were not significantly correlated with E-cadherin expression in tumor cells in the pSCC cohort (Table 2). However, the ratio of intratumoral/ stromal CD103 ${ }^{+}$TILs in pSCC was positively associated with E-cadherin expression in tumors $(P=0.021)$.

\section{Prognostic significance of $\mathrm{CD} \mathrm{C3}^{+}$and $\mathrm{CD8}^{+}$ TILs in pSCCs}

On univariate survival analysis, age, tumor size, tumor stage, and intratumoral $\mathrm{CD}_{103^{+}}$TIL numbers were associated with DFS in patients with pSCC (data not shown). Higher numbers of intratumoral CD103 ${ }^{+}$TILs

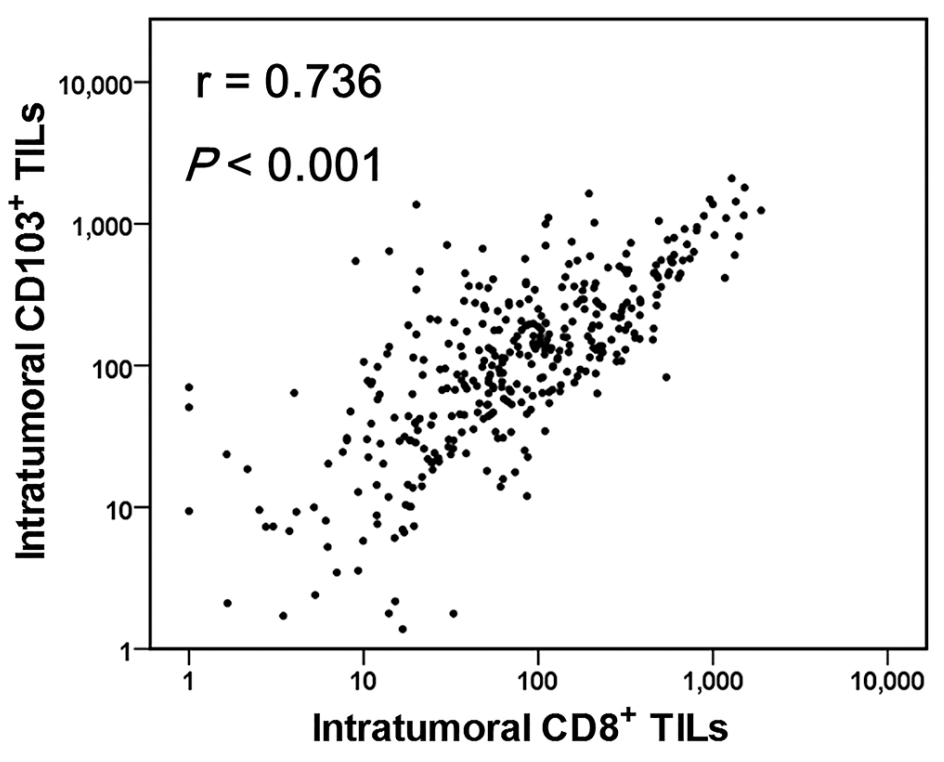

Figure 2: A strong positive correlation between the numbers of $\mathrm{CD} 103^{+}$and $\mathrm{CD8}^{+}$tumor-infiltrating lymphocytes (TILs) in pulmonary squamous cell carcinomas $(n=\mathbf{3 7 8})$. Linear regression analysis was performed to determine the relationship between $\mathrm{CD}_{103^{+}}$and $\mathrm{CD}^{+}$TIL numbers, and the correlation was analyzed using Spearman's rank correlation coefficient. 
Table 1: The numbers of intratumoral and stromal $\mathrm{CD8}^{+}$and $\mathrm{CD} 103^{+}$tumor-infiltrating lymphocytes (TILs) according to patient clinicopathological characteristics in pulmonary squamous cell carcinoma cohort $(n=378)$

\begin{tabular}{|c|c|c|c|c|c|c|c|c|c|c|}
\hline \multirow{2}{*}{\multicolumn{2}{|c|}{$\begin{array}{c}\text { Squamous cell carcinoma } \\
\begin{array}{c}\text { Clinicopathological } \\
\text { characteristics }\end{array}\end{array}$}} & \multicolumn{3}{|c|}{ Intratumoral CD103 ${ }^{+}$TILs } & \multicolumn{2}{|c|}{ Stromal CD103 ${ }^{+}$TILs } & \multicolumn{2}{|c|}{ Intratumoral $\mathrm{CD8}^{+} \mathrm{TILs}$} & \multicolumn{2}{|c|}{ Stromal CD8 ${ }^{+}$TILs } \\
\hline & & \multirow{2}{*}{$\frac{n}{360}$} & \multirow{2}{*}{$\begin{array}{c}\begin{array}{c}\text { Mean } \pm \text { SD } \\
(\text { number/mm²) }\end{array} \\
226.6 \pm 304.0\end{array}$} & \multirow{2}{*}{$\begin{array}{c}\boldsymbol{P} \\
0.206\end{array}$} & \multirow{2}{*}{$\begin{array}{c}\begin{array}{c}\text { Mean } \pm \text { SD } \\
\left(\text { number } / \mathbf{m m}^{2}\right)\end{array} \\
\frac{110.2 \pm 157.4}{}\end{array}$} & \multirow{2}{*}{$\begin{array}{c}P \\
0.222\end{array}$} & \multirow{2}{*}{$\begin{array}{c}\begin{array}{c}\text { Mean } \pm \text { SD } \\
(\text { number/mm²) }\end{array} \\
176.1 \pm 272.9\end{array}$} & \multirow{2}{*}{$\begin{array}{c}\boldsymbol{P} \\
0.718\end{array}$} & \multirow{2}{*}{$\begin{array}{c}\begin{array}{c}\text { Mean } \pm \text { SD } \\
(\text { number/mm }\end{array} \\
175.0 \pm 178.5\end{array}$} & \multirow{2}{*}{$\begin{array}{c}\boldsymbol{P} \\
0.709\end{array}$} \\
\hline Sex & male & & & & & & & & & \\
\hline & female & 18 & $135.2 \pm 146.9$ & & $64.5 \pm 85.6$ & & $151.9 \pm 163.3$ & & $135.3 \pm 87.9$ & \\
\hline \multirow[t]{2}{*}{ Age (years) } & $<60$ & 160 & $193.0 \pm 264.2$ & 0.093 & $104.0 \pm 148.8$ & 0.663 & $167.8 \pm 240.7$ & 0.656 & $161.0 \pm 144.3$ & 0.248 \\
\hline & $\geq 60$ & 218 & $243.7 \pm 320.9$ & & $111.0 \pm 159.8$ & & $180.4 \pm 288.3$ & & $182.3 \pm 195.3$ & \\
\hline \multirow[t]{2}{*}{ Smoking } & never & 22 & $154.6 \pm 240.6$ & 0.269 & $47.5 \pm 48.5$ & $<0.001$ & $110.3 \pm 146.3$ & 0.269 & $110.6 \pm 69.9$ & 0.001 \\
\hline & smoker & 342 & $227.9 \pm 304.5$ & & $114.3 \pm 160.9$ & & $177.6 \pm 276.3$ & & $178.9 \pm 181.2$ & \\
\hline \multirow[t]{2}{*}{ Tumor size } & $<5 \mathrm{~cm}$ & 284 & $239.3 \pm 322.1$ & 0.037 & $112.5 \pm 155.0$ & 0.330 & $183.2 \pm 284.9$ & 0.233 & $176.2 \pm 177.4$ & 0.570 \\
\hline & $\geq 5 \mathrm{~cm}$ & 94 & $176.7 \pm 209.3$ & & $94.5 \pm 155.2$ & & $149.8 \pm 211.2$ & & $164.1 \pm 169.9$ & \\
\hline \multirow{2}{*}{$\begin{array}{l}\text { Lymph node } \\
\text { metastasis }\end{array}$} & absent & 230 & $225.0 \pm 290.3$ & 0.852 & $97.3 \pm 133.1$ & 0.108 & $176.8 \pm 272.4$ & 0.905 & $172.2 \pm 167.4$ & 0.850 \\
\hline & present & 148 & $219.1 \pm 313.6$ & & $125.6 \pm 183.7$ & & $173.4 \pm 264.6$ & & $175.7 \pm 188.2$ & \\
\hline \multirow[t]{2}{*}{ Stage } & I/II & 298 & $229.2 \pm 311.3$ & 0.384 & $111.2 \pm 158.3$ & 0.445 & $183.0 \pm 287.8$ & 0.272 & $173.1 \pm 168.9$ & 0.982 \\
\hline & III & 80 & $196.3 \pm 247.5$ & & $96.3 \pm 142.8$ & & $145.3 \pm 178.9$ & & $173.6 \pm 199.3$ & \\
\hline
\end{tabular}

were significantly associated with prolonged DFS and OS $(P=0.021$ and 0.002 , respectively; Figure $3 \mathrm{~A})$, whereas the numbers of intratumoral $\mathrm{CD}^{+} \mathrm{TILs}$ was associated with prolonged DFS $(P=0.046$, Figure $3 \mathrm{C})$ but not OS $(P=0.113)$. The number of stromal $\mathrm{CD} 103^{+}$or $\mathrm{CD} 8^{+}$ TILs was not associated with DFS $(P=0.521$ and 0.275 , respectively) or OS ( $P=0.599$ and 0.827 , respectively; Figure 3B and 3D). Furthermore, multivariate analysis revealed that age, lymph node metastasis, and intratumoral $\mathrm{CD} 103^{+}$TILs were independent prognostic factors for DFS in pSCC (Table 3).

\section{DISCUSSION}

CD103 is expressed in various immune cells including $\mathrm{CD} 8^{+}, \mathrm{CD} 4^{+} \mathrm{T}$ cells, natural killer cells, natural killer-like $\mathrm{T}$ cells, as well as mast cells and dendritic cells $[18,21,24]$. Furthermore, $\mathrm{CD}^{+} \mathrm{CD} 103^{+}$TILs showed features characteristic of tissue-resident memory $\mathrm{T}$ cells that exhibit tumor-specific cytolytic abilities upon stimulation [20]. These findings suggest that CD103+ TILs are critically involved in tumor surveillance. Our study demonstrated that a high number of intratumoral $\mathrm{CD} 103^{+}$ cells was associated with a favorable prognosis in patients with pSCC but not pADC. Consistent with our results, CD103 expression was observed in $10-30 \%$ of $\mathrm{CD} 8^{+}$ TILs isolated from NSCLC tissues [20]. Moreover, high numbers of $\mathrm{CD} 103^{+}$cells among intratumoral $\mathrm{CD} 8^{+} \mathrm{T}$ cells have been associated with more favorable prognoses in patients with ovarian and bladder cancers [18, 22]. Based on these results and those from our study, we suggest that the infiltration of $\mathrm{CD} 8^{+} \mathrm{CD} 103^{+}$TILs is also a predictor of very favorable prognoses in patients with $\mathrm{pSCC}$, as well as those with ovarian and bladder cancers. Crosstalk between cells in intratumoral and stromal regions might be critical for tumor surveillance, suggesting that investigating CD103 expression patterns in these two tumor regions may enable a more comprehensive understanding of the tumor microenvironment. In this study, the preferential expression of CD103 and CD8 in the immune cells of intratumoral rather than stromal regions was associated with a good prognosis in patients with pSCC. Previously, we reported that $\mathrm{CD} 8^{+}$TILs were associated with the expression of PD-1 pathway-related molecules and a better prognosis in patients with pSCC [25], similar to other types of cancers [9-12]. Nevertheless, multivariate survival analysis revealed that the number of intratumoral $\mathrm{CD}_{103^{+}}$TILs is a more powerful predictor of patient survival than the number of CD8 ${ }^{+}$TILs in $\mathrm{pSCC}$.

Meanwhile, tumor-infiltrating regulatory $\mathrm{T}$ cell (Treg) also expresses CD103 [26, 27]. To address this, we evaluated the intratumoral and stromal Tregs using immunohistochemistry for FoxP3 (a representative marker of Treg) in pSCC cohort. The numbers of FoxP3 ${ }^{+}$TILs in intratumoral and stromal regions were $18.4 \pm 31.6$ and $37.7 \pm 44.6$, respectively (data not shown). The quantity and distribution pattern of Tregs were different from those of CD103+ TILs (intratumoral, $222.2 \pm 299.0$; stromal, $108.7 \pm 185.0$ ) and CD $8^{+}$TILs (intratumoral, $175.0 \pm 268.8$; stromal, $173.2 \pm 175.4)$. Accordingly, the correlation coefficient between intratumoral CD $103^{+}$TILs and FoxP3 $^{+}$ TILs was much lower than that between intratumoral $\mathrm{CD}_{103^{+}}$TILs and CD8 ${ }^{+}$TILs (0.174 and 0.736, respectively). Consistent with our data, a small proportion of Tregs co-expressed CD103 [27] and the percentage of $\mathrm{CD}^{+} \mathrm{CD} 103^{+}$TILs was much lower than $\mathrm{CD} 8^{+} \mathrm{CD} 103^{+}$ TILs in patients with ovarian and bladder cancers $[18,22]$. The number of intratumoral and stromal FoxP3 ${ }^{+}$TILs was not significantly associated with DFS and OS of patients with $\mathrm{pSCC}$ in this study (data not shown). Based on those 
Table 2: The number of CD103 ${ }^{+}$TILs and the ratio of intratumoral/stromal CD103 ${ }^{+}$TILs according to E-cadherin expression in pulmonary squamous cell carcinoma cohort

\begin{tabular}{|c|c|c|c|c|c|c|c|c|}
\hline & & \multicolumn{3}{|c|}{$\begin{array}{c}\text { Intratumoral } \mathrm{CD} \mathrm{103}^{+} \\
\text {TILs }\end{array}$} & \multicolumn{2}{|c|}{ Stromal CD103 ${ }^{+}$TILs } & \multicolumn{2}{|c|}{$\begin{array}{c}\text { Intratumoral/stromal } \\
\mathrm{CD}{ }^{+} \text {TIL ratio }\end{array}$} \\
\hline & & $n$ & $\begin{array}{c}\text { Mean } \pm \text { SD } \\
\left(\text { number } / \mathbf{m m}^{2}\right)\end{array}$ & $P$ & $\begin{array}{c}\text { Mean } \pm \text { SD } \\
\left(\text { number } / \mathbf{m m}^{2}\right)\end{array}$ & $P$ & Mean & $P$ \\
\hline E-cadherin & Negative & 97 & $228.6 \pm 296.1$ & 0.787 & $151.7 \pm 198.3$ & 0.001 & 3.2 & 0.021 \\
\hline expression & Positive & 250 & $218.7 \pm 307.5$ & & $80.7 \pm 121.2$ & & 4.9 & \\
\hline
\end{tabular}

Abbreviations: TILs, tumor infiltrating lymphocytes; SD, standard deviation.

Table 3: Multivariate analysis of disease-free survival outcomes in pulmonary squamous cell carcinoma cohort

\begin{tabular}{|c|c|c|c|}
\hline & Odds ratio & $95 \%$ confidence interval & $P$ value \\
\hline \multicolumn{4}{|l|}{ Disease-free survival } \\
\hline Age & 2.223 & $1.576-3.138$ & $<0.001$ \\
\hline Lymph node metastasis & 1.551 & $1.122-2.144$ & 0.008 \\
\hline Intratumoral $\mathrm{CD} 103^{+} \mathrm{TILs}$ & 0.648 & $0.449-0.935$ & 0.021 \\
\hline Stromal CD $103^{+}$TILs & 1.040 & $0.678-1.593$ & 0.859 \\
\hline Intratumoral $\mathrm{CD}^{+}{ }^{+} \mathrm{TILs}$ & 0.953 & $0.643-1.413$ & 0.810 \\
\hline Stromal CD8 ${ }^{+}$TILs & 0.867 & $0.542-1.386$ & 0.551 \\
\hline
\end{tabular}

Abbreviations: TILs, tumor-infiltrating lymphocytes.
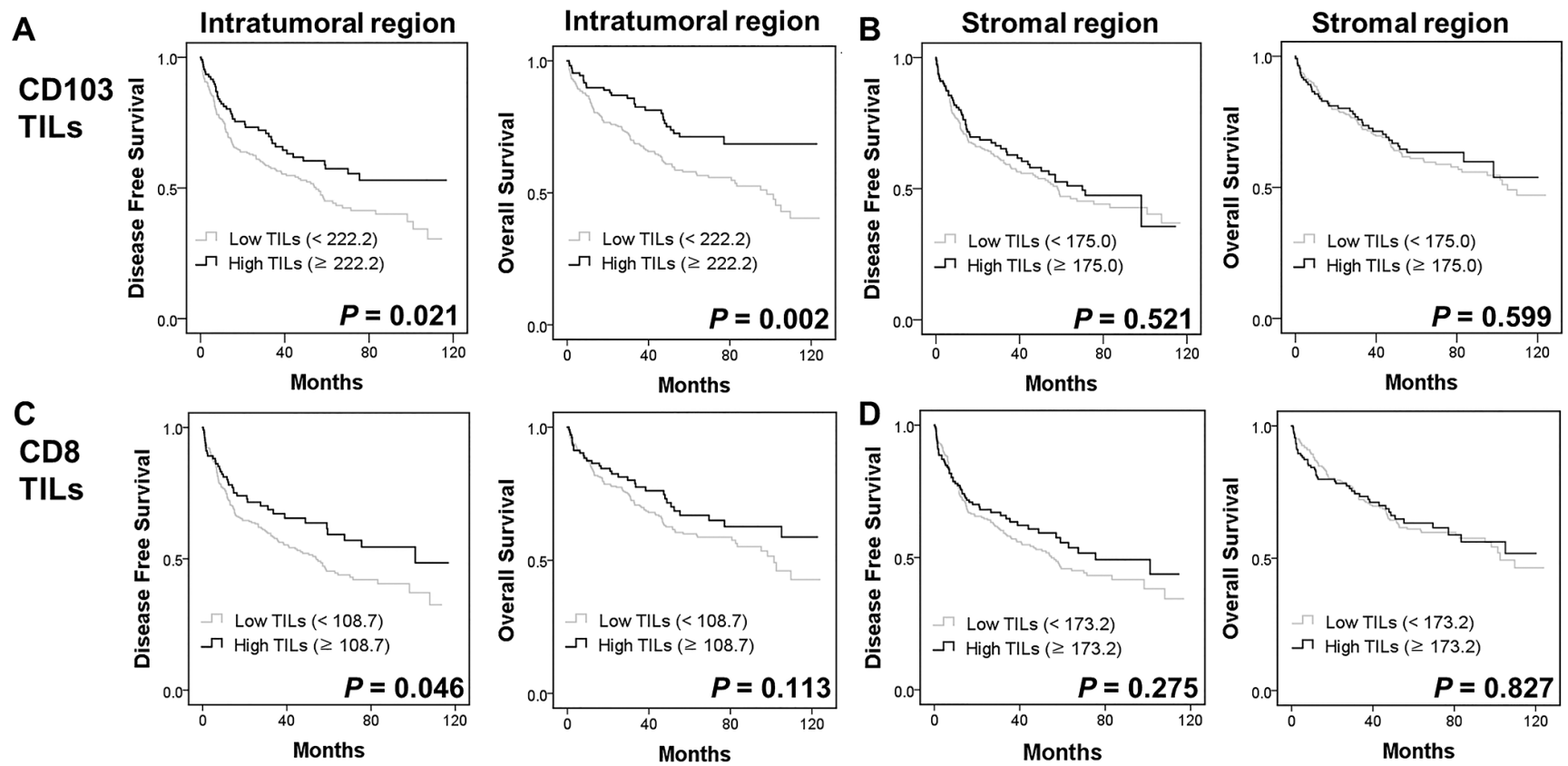

Figure 3: Kaplan-Meier plots using the log rank test for disease-free survival (DFS) and overall survival (OS) in patients with pulmonary squamous cell carcinoma $(n=378)$ according to intratumoral and stromal $\mathrm{CD}_{103^{+}}(\mathbf{A}, \mathbf{B})$, and CD $8^{+} \mathrm{TIL}$ numbers $(\mathbf{C}, \mathbf{D})$. 
previous studies and our present study, it is suggested that the favorable prognostic implication of high intratumoral $\mathrm{CD} 103^{+}$TILs may be attributable to the $\mathrm{CD} 8^{+} \mathrm{CD} 103^{+}$TILs

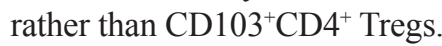

In this study, a significant prognostic implication of CD103 expression in TILs was observed in SCC but not in ADC subtype of NSCLC. A high number of intratumoral $\mathrm{CD}_{103^{+}}$TILs was related to improved survival in pSCC. In several studies, the interaction between CD103 and E-cadherin increased granule polarization and exocytosis in TILs [13, 14] and enhanced recruitment and retention of tumor-antigen specific TILs, in ovarian serous carcinoma, glioma, and NSCLC [17-19]. In our study, the H-score and positivity of E-cadherin expression were much higher in pSCC than in pADC. Moreover, a significantly higher ratio of intratumoral/stromal $\mathrm{CD} 103^{+}$TILs was observed in pSCC expressing high levels compared to low levels of E-cadherin. Based on these findings, it is feasible that the interaction between CD103 expressed in TILs and E-cadherin expressed in tumor cells might affect tumor immune surveillance and clinical outcomes in patients with pSCC.

Recently, PD-1/PD-L1-targeted immunotherapy was approved by the U.S. Food Drug Administration for the treatment of patients with advanced NSCLC and was deemed a highly promising and safe therapy for cancer [7]. PD-1 expression is induced in T cells that are chronically stimulated by antigens, including viral and tumor antigens [7]. Thus, it is feasible that $\mathrm{CD} 103^{+} \mathrm{T}$ cells entrapped in the intratumoral (i.e., intraepithelial) compartment are exhausted by chronic tumor antigen stimulation, thereby up-regulating PD-1 [28]. Consistent with this suggestion, we observed a strong positive correlation between the number of PD- $1^{+}$and CD103 ${ }^{+}$TILs in $\mathrm{pSCC}(r=0.449, P$ $<0.001$, data not shown). Moreover, $\mathrm{CD}^{+} \mathrm{CD} 103^{+}$TILs display a tumor-specific, tissue-resident memory $\mathrm{T}$ cell phenotype and frequently express immune checkpoint molecules including PD-1 and Tim-3 [20]. Taken together, we suggest that intratumoral CD $103^{+}$TILs may serve as a predictive biomarker for PD-1/PD-L1-targeted immunotherapy in NSCLC, as previously proposed [28].

The favorable prognostic implication of high $\mathrm{CD}_{103^{+}}$ TILs and the positive correlation between $\mathrm{CD} 103^{+}$and PD- $1^{+}$TILs might seem to be conflicting, considering that PD-1 is a maker of exhausted T cells. Our and other groups demonstrated that a high number of PD-1 TILs was associated with better prognosis in patients with pADC, pSCC and head and neck SCC [12, 25, 29]. It was suggested that increased PD- $1^{+}$TILs may reflect a preexisting immune response against tumor cells, thus being associated with favorable prognosis of patients with tumor.

Meanwhile, the so-called "immunoscore" has been established as a prognostic biomarker for anti-tumor immune responses [30]. This has been confirmed by many studies using various methods [12, 25, 31, 32]. Among these methods, IHC has emerged as a powerful technology to predict tumor progression and patient survival. However, this method needs to be improved, because interpretation of IHC results is often subjective and dependent on the interpreter. To overcome this limitation, we interpreted IHC results by computer program-based image analysis, which often provides an objective and reproducible interpretation, although standard guidelines need to be established further.

In conclusion, this study suggests that intratumoral $\mathrm{CD}_{103}{ }^{+}$TILs represent an important prognostic biomarker for predicting better survival in patients with $\mathrm{pSCC}$, and as such, may be a potential target candidate for the development of cancer immunotherapies.

\section{MATERIALS AND METHODS}

\section{Patients and samples}

In this study, we established two cohorts: patients with NSCLC and those with pSCC specifically. For the NSCLC cohort, 132 consecutive patients with pADC $(n=85)$ and pSCC $(n=47)$, who underwent resection at Seoul National University Hospital (SNUH), were retrospectively evaluated for the potential prognostic value of CD $103^{+}$TILs in NSCLC. For the pSCC cohort, 378 patients with pSCC who underwent surgery and had been followed up at SNUH were evaluated to validate the findings in the NSCLC cohort. We excluded those patients who had received chemotherapy before surgery or those who had distant metastasis at the time of diagnosis. Clinicopathologic data and the pathologic tumor-nodemetastasis stage according to the 7th American Joint Committee on Cancer were obtained from medical and pathological records. A tissue microarray was constructed from $2 \mathrm{~mm}$ diameter cores derived from representative tumor regions of formalin-fixed paraffin-embedded tissue blocks. This study was performed in accordance with the recommendations of the World Medical Association Declaration of Helsinki and was approved by the Institutional Review Board of SNUH (H-1404-100-572).

\section{Immunohistochemistry}

IHC for CD103 was performed using a rabbit monoclonal CD103 antibody (Abcam; Cambridge, UK) and the Benchmark XT autostainer (Ventana Medical Systems; Tucson, AZ, USA). IHC for CD8 (rabbit IgG, clone SP16, Thermo Scientific Fischer; Rockford, IL, USA), Foxp3 (Ab20034, Abcam; Cambridge, UK) and E-cadherin (clone 36B5, Novocastra; New Castle, UK) was performed using the Bond-Max automated immunostainer (Leica Microsystems; Melbourne, Australia). E-cadherin IHC results were evaluated based on staining intensity in tumor cell membranes and scored as follows: : 0, negative; 1 , weak or moderate in $\leq 5 \%$ of tumor cells; 2 , moderate in $\geq 5 \%$ of tumor cells; 3 , strong in $\geq 5 \%$ of tumor cells. A score of 2 or 3 was considered positive for E-cadherin 
expression. H-score was calculated using the formula, the representative staining intensity of each case $\times$ the percentage of expressed tumor cells.

\section{Automated quantification of $\mathrm{CD} \mathrm{C3}^{+}, \mathrm{CD8}^{+}$and FoxP3 $^{+}$TILs}

Automated counting of TILs on IHC slides was performed as previously reported.[12, 25] Briefly, slides stained for CD8, CD103 and FoxP3 were scanned using the Aperio ScanScope (Aperio Technologies; Vista, CA, USA), and automated counting was performed using modified nuclear algorithms for IHC in the Aperio ImageScope software (Aperio Technologies). The numbers of intratumoral and stromal $\mathrm{CD} 103^{+}, \mathrm{CD}^{+}$and FoxP3 $3^{+}$TILs per unit area $\left(\mathrm{mm}^{2}\right)$ were calculated and used for statistical analyses.

\section{Statistical analysis}

Quantitative data with a normal distribution are presented as means \pm standard deviation. All of the statistical analyses were performed using SPSS software (version 23; IBM Corp., New York, NY, USA). Comparisons between variables were performed using the Student's $t$-test. Cut-off intratumoral or stromal CD103, $\mathrm{CD}^{+}$and $\mathrm{FoxP}^{+}{ }^{+} \mathrm{TIL}$ numbers were determined based on the means for categorical analyses. DFS was measured from the date of surgery to that of recurrent or metastatic disease occurrence. OS was measured from the date of diagnosis to that of death from any cause. Survival analysis was performed using the Kaplan-Meier method with the log-rank test. Multivariate Cox regression analysis was performed with consideration of co-linearity. Two-sided $P$ values $<0.05$ were considered statistically significant.

\section{ACKNOWLEDGMENTS AND FUNDING}

This research was supported by the Basic Science Research Program (grant No. NRF2016R1D1A1B01015964) through the National Research Foundation (NRF) funded by the Ministry of Education, Science and Technology (MEST) and a grant (No. HI14C0069) of the Korea Health Technology R\&D Project through the Korea Health Industry Development Institute (KHIDI) funded by the Ministry of Health \& Welfare, Republic of Korea.

\section{CONFLICTS OF INTEREST}

No potential conflicts of interest were disclosed by the authors.

\section{REFERENCES}

1. Lindeman NI, Cagle PT, Beasley MB, Chitale DA, Dacic S, Giaccone G, Jenkins RB, Kwiatkowski DJ, Saldivar JS,
Squire J, Thunnissen E, Ladanyi M. College of American Pathologists International Association for the Study of Lung $\mathrm{C}$ and Association for Molecular P. Molecular testing guideline for selection of lung cancer patients for EGFR and ALK tyrosine kinase inhibitors: guideline from the College of American Pathologists, International Association for the Study of Lung Cancer, and Association for Molecular Pathology. J Mol Diagn. 2013; 15:415-453.

2. Steuer CE, Ramalingam SS. ALK-positive non-small cell lung cancer: mechanisms of resistance and emerging treatment options. Cancer. 2014; 120:2392-2402.

3. Ohashi K, Maruvka YE, Michor F, Pao W. Epidermal growth factor receptor tyrosine kinase inhibitor-resistant disease. J Clin Oncol. 2013; 31:1070-1080.

4. Kim S, Kim TM, Kim DW, Go H, Keam B, Lee SH, Ku JL, Chung DH, Heo DS. Heterogeneity of genetic changes associated with acquired crizotinib resistance in ALKrearranged lung cancer. J Thorac Oncol. 2013; 8:415-422.

5. Albini A, Sporn MB. The tumour microenvironment as a target for chemoprevention. Nat Rev Cancer. 2007; 7:139-147.

6. Kerkar SP, Restifo NP. Cellular constituents of immune escape within the tumor microenvironment. Cancer Res. 2012; 72:3125-3130.

7. Topalian SL, Drake CG, Pardoll DM. Immune checkpoint blockade: a common denominator approach to cancer therapy. Cancer Cell. 2015; 27:450-461.

8. Apetoh L, Smyth MJ, Drake CG, Abastado JP, Apte RN, Ayyoub M, Blay JY, Bonneville M, Butterfield LH, Caignard A, Castelli C, Cavallo F, Celis E, et al. Consensus nomenclature for CD8 $\mathrm{T}$ cell phenotypes in cancer. Oncoimmunology. 2015; 4:e998538.

9. Fu J, Xu D, Liu Z, Shi M, Zhao P, Fu B, Zhang Z, Yang H, Zhang H, Zhou C, Yao J, Jin L, Wang H, et al. Increased regulatory $\mathrm{T}$ cells correlate with $\mathrm{CD} 8 \mathrm{~T}$-cell impairment and poor survival in hepatocellular carcinoma patients. Gastroenterology. 2007; 132:2328-2339.

10. Sato E, Olson SH, Ahn J, Bundy B, Nishikawa H, Qian F, Jungbluth AA, Frosina D, Gnjatic S, Ambrosone C, Kepner J, Odunsi T, Ritter G, et al. Intraepithelial CD8+ tumor-infiltrating lymphocytes and a high $\mathrm{CD} 8+$ /regulatory $\mathrm{T}$ cell ratio are associated with favorable prognosis in ovarian cancer. Proc Natl Acad Sci USA. 2005; 102:18538-18543.

11. Sharma P, Shen Y, Wen S, Yamada S, Jungbluth AA, Gnjatic S, Bajorin DF, Reuter VE, Herr H, Old LJ, Sato E. CD8 tumor-infiltrating lymphocytes are predictive of survival in muscle-invasive urothelial carcinoma. Proc Natl Acad Sci USA. 2007; 104:3967-3972.

12. Koh J, Go H, Keam B, Kim MY, Nam SJ, Kim TM, Lee SH, Min HS, Kim YT, Kim DW, Jeon YK, Chung DH. Clinicopathologic analysis of programmed cell death-1 and programmed cell death-ligand 1 and 2 expressions in pulmonary adenocarcinoma: comparison with histology and driver oncogenic alteration status. Mod Pathol. 2015; 28:1154-1166. 
13. Franciszkiewicz K, Le Floc'h A, Boutet M, Vergnon I, Schmitt A, Mami-Chouaib F. CD103 or LFA-1 engagement at the immune synapse between cytotoxic $\mathrm{T}$ cells and tumor cells promotes maturation and regulates T-cell effector functions. Cancer Res. 2013; 73:617-628.

14. Le Floc'h A, Jalil A, Vergnon I, Le Maux Chansac B, Lazar V, Bismuth G, Chouaib S, Mami-Chouaib F. Alpha $\mathrm{E}$ beta 7 integrin interaction with E-cadherin promotes antitumor CTL activity by triggering lytic granule polarization and exocytosis. J Exp Med. 2007; 204:559-570.

15. Agace WW, Higgins JM, Sadasivan B, Brenner MB, Parker CM. T-lymphocyte-epithelial-cell interactions: integrin alpha(E)(CD103)beta, LEEP-CAM and chemokines. Curr Opin Cell Biol. 2000; 12:563-568.

16. Mueller SN, Gebhardt T, Carbone FR, Heath WR. Memory $\mathrm{T}$ cell subsets, migration patterns, and tissue residence. Annu Rev Immunol. 2013; 31:137-161.

17. Jouanneau E, Black KL, Veiga L, Cordner R, Goverdhana S, Zhai Y, Zhang XX, Panwar A, Mardiros A, Wang H, Gragg A, Zandian M, Irvin DK, et al. Intrinsically desialylated CD103(+) CD8 T cells mediate beneficial antiglioma immune responses. Cancer Immunol Immunother. 2014; 63:911-924.

18. Webb JR, Milne K, Watson P, Deleeuw RJ, Nelson BH. Tumor-infiltrating lymphocytes expressing the tissue resident memory marker CD103 are associated with increased survival in high-grade serous ovarian cancer. Clin Cancer Res. 2014; 20:434-444.

19. Zikos TA, Donnenberg AD, Landreneau RJ, Luketich JD, Donnenberg VS. Lung T-cell subset composition at the time of surgical resection is a prognostic indicator in nonsmall cell lung cancer. Cancer Immunol Immunother. 2011; 60:819-827.

20. Djenidi F, Adam J, Goubar A, Durgeau A, Meurice G, de Montpreville V, Validire P, Besse B, Mami-Chouaib F. CD8+CD103+ tumor-infiltrating lymphocytes are tumorspecific tissue-resident memory $\mathrm{T}$ cells and a prognostic factor for survival in lung cancer patients. J Immunol. 2015; 194:3475-3486.

21. Quinn E, Hawkins N, Yip YL, Suter C, Ward R. CD103+ intraepithelial lymphocytes - a unique population in microsatellite unstable sporadic colorectal cancer. Eur J Cancer. 2003; 39:469-475.

22. Wang B, Wu S, Zeng H, Liu Z, Dong W, He W, Chen X, Dong X, Zheng L, Lin T, Huang J. CD103+ Tumor
Infiltrating Lymphocytes Predict a Favorable Prognosis in Urothelial Cell Carcinoma of the Bladder. J Urol. 2015; 194:556-562.

23. Zhang N, Bevan MJ. Transforming growth factor-beta signaling controls the formation and maintenance of gutresident memory $\mathrm{T}$ cells by regulating migration and retention. Immunity. 2013; 39:687-696.

24. Sung SS, Fu SM, Rose CE, Jr., Gaskin F, Ju ST, Beaty SR. A major lung CD103 (alphaE)-beta7 integrin-positive epithelial dendritic cell population expressing Langerin and tight junction proteins. J Immunol. 2006; 176:2161-2172.

25. Kim MY, Koh J, Kim S, Go H, Jeon YK, Chung DH. Clinicopathological analysis of PD-L1 and PD-L2 expression in pulmonary squamous cell carcinoma: Comparison with tumor-infiltrating T cells and the status of oncogenic drivers. Lung Cancer. 2015; 88:24-33.

26. Anz D, Mueller W, Golic M, Kunz WG, Rapp M, Koelzer VH, Ellermeier J, Ellwart JW, Schnurr M, Bourquin C, Endres S. CD103 is a hallmark of tumor-infiltrating regulatory T cells. Int J Cancer. 2011; 129:2417-2426.

27. Allakhverdi Z, Fitzpatrick D, Boisvert A, Baba N, Bouguermouh S, Sarfati M, Delespesse G. Expression of CD103 identifies human regulatory T-cell subsets. J Allergy Clin Immunol. 2006; 118:1342-1349.

28. Webb JR, Milne K, Nelson BH. Location, location, location: CD103 demarcates intraepithelial, prognostically favorable CD8 tumor-infiltrating lymphocytes in ovarian cancer. Oncoimmunology. 2014; 3:e27668.

29. Badoual C, Hans S, Merillon N, Van Ryswick C, Ravel P, Benhamouda N, Levionnois E, Nizard M, Si-Mohamed A, Besnier N, Gey A, Rotem-Yehudar R, Pere H, T et al. $\mathrm{PD}$-1-expressing tumor-infiltrating $\mathrm{T}$ cells are a favorable prognostic biomarker in HPV-associated head and neck cancer. Cancer Res. 2013; 73:128-138.

30. Angell H, Galon J. From the immune contexture to the Immunoscore: the role of prognostic and predictive immune markers in cancer. Curr Opin Immunol. 2013; 25:261-267.

31. Liu S, Lachapelle J, Leung S, Gao D, Foulkes WD, Nielsen TO. CD8+ lymphocyte infiltration is an independent favorable prognostic indicator in basal-like breast cancer. Breast Cancer Res. 2012; 14:R48.

32. Mahmoud SM, Paish EC, Powe DG, Macmillan RD, Grainge MJ, Lee AH, Ellis IO, Green AR. Tumorinfiltrating CD8+ lymphocytes predict clinical outcome in breast cancer. J Clin Oncol. 2011; 29:1949-1955. 